\title{
Research on the Modern Intelligent Healthcare Platform from the Perspectives of Grid based Cloud Computing and Information Management System Assisted Internet of Things Technology
}

\author{
Nan Feng* \\ Department of Health Management, Xi'an Medical University, \\ $X i^{\prime}$ an 710021, China \\ fengnanxa@sina.com
}

\begin{abstract}
In this paper, we research on the modern intelligent healthcare platform from the perspectives of grid based cloud computing and information management system assisted Internet of things technology. In the increasing pressure of modern society, people often attend the poor general health. The more deadly is that people lack the necessary technical means that our physical condition of the related data. Hospital information construction is to adapt to the information age and the modern medical mode, necessary measures of health economy and the hospital information system is the inevitable outcome of the application of information technology in hospital. Excellent hospital information system for standardized hospital management, and security of clinical activities smoothly to carry out the relevant scientific research activity has the important meaning. Intelligent medical treatment is the use of advanced network, communication, computer and digital technology, to implement the intelligent of the medical information collection, conversion, storage, transmission and processing, and various medical business process of digital operation, thus realizes the patients and medical staff, medical institutions, medical equipment, the interaction between, gradually achieve the medical information. Intelligent medical treatment can not only improve the hospital and medical staff's working efficiency, reduce mistakes in the work, also can through the remote medical treatment, remote consultation and so on ways to solve the problem of unequal distribution of regional health resources. Our research combines the grid and cloud computing technique to build up the novel intelligent healthcare platform with IOT technique which will enhance the current medical treatment mode and level up the overall performance of the modern information management system for the general usages.
\end{abstract}

Keywords: Healthcare Platform; Grid Computing; Cloud based Computing; Information Management; Internet of Things; Data Analysis and Knowledge Discovery

\section{Introduction}

From treatment to prevention, it is a trend in the development of modern medicine. In the increasing pressure of modern society, people often attend the poor general health. The more deadly is that people lack the necessary technical means that our physical condition of the related data. Most people are in ignorance, no sleep or in hindsight captured by the disease. ${ }^{1}$

People look from the existing means of prevention but the disease prevention measures is still confined to perfect and proper diet, regular life entertainment such as basic means, a higher level of real-time monitoring of blood pressure, heart rate and so on are not their own can do it. Intelligent healthcare is the Internet of things technology used in medical field, with the aid of digitalization and visualization model, for the collection

Nan Feng is the corresponding author. 
and health monitoring of the vital signs will let more people share the limited medical resources and the health also is wisdom. Wisdom medical scheme can make rescue more efficient especially for the patients with heart disease and the treatment time is critical. There have been some heart disease patients carry the special wireless terminal equipment and the equipment can continuously through wireless health IOT sends data real-time data and other signs to medical monitoring center. For those who often forget to take medicine amnesiacs or old man, it will find the package is a good helper which can send medication reminders for the patients in the timely manner and easily to provide drug information database which will be meaningful and necessary [1-3].

In general, the modern intelligent healthcare platform could be generally made up of the following parts. (1) Infrastructure platform. Infrastructure platform mainly consists of wisdom perception layer and healthcare private network. Among them, the wisdom of medical health perception layer involves different kinds of sensors and sensor gateway which will realize the recognition of object of medical and health care and medical and health resources collection. (2) Based support system. Based support system is mainly composed of the operation support platform and infrastructure. Operation support platform in position of the flow is composed of two parts: one is based on the middleware which provide resource virtualization, middleware, application service middleware, database middleware. Second is operation support services, through the downward to realize the organic integration of infrastructure to provide the cloud computing and cloud storage capabilities to solve the centralized management of scattered resources and pooling resources scattered service problems, effectively support all kinds of resources and data to achieve service-oriented perception of on-demand based application aggregation and support efficient analysis of massive data processing. (3) The basic standard specification system. Standard system is foundation of the wisdom medical construction work and also is the basic premise of information exchange and sharing. Wisdom cloud data center was established in the unity of the core data framework, under the premise of construction is carried out based on national standard can complete convergence of medical institutions relevant information integration and support the residents' health information sharing [4-7].

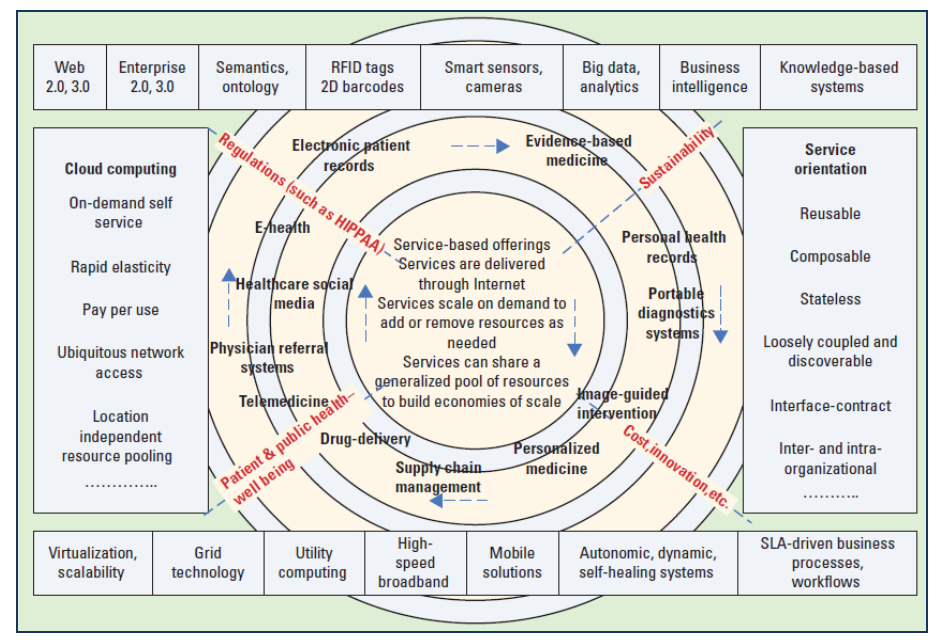

\section{Figure 1. The Modern Cloud Intelligent Healthcare Platform Structure}

As illustrated in the above figure one, the modern intelligent healthcare platform uses the following computer based techniques to help modify the system. (1) Cloud computing tool. Cloud computing is grid computing, distributed computing, network storage, virtualization and other traditional fusion product of computer and network technology development, also is a kind of emerging shared infrastructure. In the medical industry of 
cloud computing, the patient's electronic medical record or inspection information is stored in the central server, patients can access to global information and related information, medical staff real-time access to information from the Internet activation device. (2) The data mining and integration technology. Data fusion technology is to point to make full use of multi-sensor information in different time and space, by using computer to the time sequence for a number of observation information, under a certain criterion to automatic analysis, comprehensive, dominance and use of the object to be measured for the consistency of interpretation and description, in order to complete the required tasks for the decision-making and the evaluation of information processing technology. Originating in multi-sensor target information of different time or the same time the target information more comprehensive processing, coordination optimization, greatly improve the intellectualization and information level of medical treatment system. (3) Mobile computing technology. Mobile computing technology refers to the mobile terminal through wireless communications and other mobile terminal information interaction or fixed computing devices have a purpose. Mobile computing to the far of moving objects detection and early warning and support the rapid transmission of data, for the medical staff of first aid to win time [8-15]. (4) The Internet of things technology. In the medical and health field, the main applications of the Internet of things technology lies in the material management process visualization technology, digital medical based information technology and medical digital technology from three aspects.

Below the modern intelligent healthcare platform, the medical information management system (MIS) serves as the foundation for the whole system. With the coming of information assist age and the rapid development of medical and health undertakings, the hospital is an increasing amount of information processing, the general relationship between information and complicated, so the requirements from the perspective of whole hospital information processing, analysis and partial information form and contact is urgently needed. In the Figure 2, we illustrate the primary structure of the information management system model.

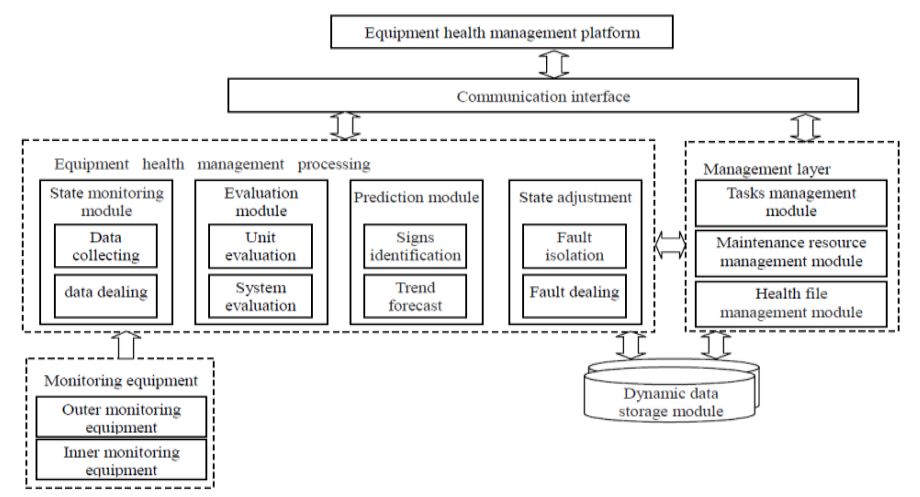

\section{Figure 2. The Primary Structure of the Information Management System Model}

As far as the mentioned system is concerned, the traditional MIS system for the medical usages could be separated into the following decompositions. System design in detail the maintenance management of process, equipment, application department through the hospital internal office network input equipment maintenance application form, the real-time large screen display equipment maintenance department and each link maintenance for follow-up. Measuring equipment basic information management module includes measuring equipment and measuring equipment calibration information, to ensure that the system can quickly query the metrological verification of each measurement devices, including the number of qualified, verification department and verification personnel information. According to the general set of metrological 
verification plan, after entering management module, the system will appear in the near future to complete the verification of the measurement equipment list, facilitate verification personnel to make corresponding arrangements. Medical equipment archives management in the form of completely by hand before, such as incomplete file incomplete collection, sorting and find many problems such as the difficult and the easy loss [16-18]. This system will largely enhance the efficiency of the modern medical service.

In this paper, we conduct theoretical research on the modern intelligent healthcare platform from the perspectives of grid based cloud computing and information management system assisted Internet of things technology. For development of hospital information construction and the users, can make full use of current resources and advanced technology, flexible from cloud service providers to retrieve the required hardware and software resources, reduce the cost information for software and hardware resources and the cost of the daily information management. Hospital only need to spend more energy and money on the medical equipment and medical technology, all-round to improve the level of medical treatment to provide better medical service for more patients. We will discuss the problems in-depth later.

\section{The Cloud Computing System and Data Analysis and Transmission}

\subsection{The Data Mining and Storage for the Cloud System}

The rapid development of database technology to make information technology permeates into multiple areas, including health care. Medical field there are a large number of data, such as the patient's medical history, the doctor daily diagnosed records, inspection reports, clinical treatment, drug use, etc. As regional health information platform in medical health database is gradually expanding, the scale expands gradually, its complexity is increasing. Although data accumulated a large number of medical health records, but rarely come out something of value in the data mining on various kinds of health care decisions throughout the day. The establishment of the medical health records can not only from the auxiliary disease prevention and control of management, and it also implies some information associated with the disease. We firstly define the basic formulas as the follows.

$$
\begin{aligned}
& R^{\times}(X)=\{Y \mid(Y \in U / \operatorname{IND}(R)) \cap(Y \in X)\} \\
& R_{\times}(X)=\{Y \mid(Y \in U / \operatorname{IND}(R)) \cap(Y \cap X \neq \varnothing)\} \\
& \lambda(X, Y)=\operatorname{Card}(\operatorname{POS}(X, Y)) / \operatorname{Card}(U)
\end{aligned}
$$

In the formula one and two, we define the lower and upper approximation, respectively. In the formula three, we define the similarity measurement standard. Compared with traditional data mining algorithms, we use the Bayesian theory based algorithm to undertake the tasks. Because the model space is too big, measure is calculated for each model is almost impossible, so this kind of method generally uses heuristic search algorithm, namely through a heuristic function to choose the next step in the current search node, usually is to choose the minimum cost. In order to reduce the search space, this kind of method generally requires first of all sort of Bayesian network node. Algorithm executes usually begin with an empty diagram, through a certain search strategy to find an edge, and add to the diagram. If so, then adding the side figure and keep the same steps until no new and novel figure is better than the old. In the formula 4, we define the criteria function. 
$\operatorname{Information}(X, Y)=\sum_{j=1}^{k} \frac{y_{1 j}+y_{2 j}+\ldots+y_{m j}}{y} \operatorname{Info}\left(y_{1 j}, y_{2 j}, \ldots, y_{m j}\right)$

To enhance the performance of the traditional criteria function, we add the modified fuzzy parameter into the function which is defined as the follows.

$R X=\left\{x \mid[x]_{R} \subseteq X, x \in U\right\}$

Medical data in a distributed retrieval behavior include tracking medical packets, change the packet content and change the ID, forwarding packets. Considering the medical data can be detected and computability, query flabby ideas focusing on medical packet number, data content repetition rate, neighborhood node data similarity and correlation to analyze data distribution behavior, medical data query [19]. The formula $6 \sim 7$ shows the definition.

$$
\begin{aligned}
& P(X \mid Y)=\frac{P(X, Y)}{P(Y)} \\
& P(X \mid Y)=P(X) \prod_{i=1}^{n} \prod_{j=1}^{q} \frac{\left(\gamma_{i}-1\right) !}{\left(N_{i j}+\gamma_{i}-1\right)} \prod_{k=1}^{\gamma} N_{i j k}
\end{aligned}
$$

Grading a greedy algorithm is a kind of strategy, its basic thought proceeds from an initial state of the problem, in each step according to some greedy strategy always made in the current view is the best option and makes sure that every step to get a local optimal solution. In each step according to the conditions of the local optimization choose a data, if the data and the local optimal solution together are feasible, the data will be added to the current local optimal solution to generate a new local optimal solution. Iteration the process until all data after exhaustive or couldn't be better local optimal solution. In the next Figure 3, we show the flowchart of the proposed data analysis algorithm for the system.

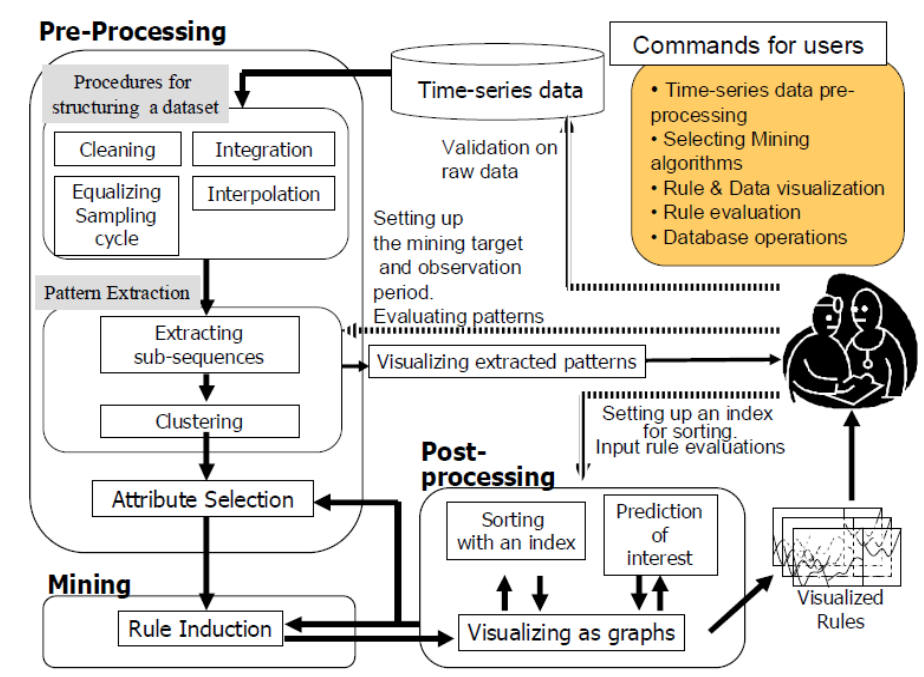

Figure 3. The Flowchart of the Proposed Data Analysis Algorithm for the System 


\subsection{The Cloud Computing System Architecture}

Cloud data center contains large number of computers. Operating costs are high. Effective integration of resources, improve resource utilization efficiency, save energy, reduce running cost is the hot spot in the cloud data center. The cloud data center through virtualization technology will computing resources, storage resources and network resources to build into a dynamic virtual resource pool and the use of virtual resource management techniques to realize automatic cloud computing resources deployment to each according to his need. The cloud computing technique could be separated into three layers. (1) IaaS. The cloud platform service providers in the data center, infrastructure hardware resources, such as virtual host, storage way provided to users via the Internet. For users, they do not need to purchase any hardware information system operation, only need to rent these according to the actual demand of the Settings to get the required computing power and storage capacity. Using this model, the user can need according to the temporary emergency information to purchase infrastructure services, hardware realization of hospital information development service outsourcing, reduce the hardware cost of investment. (2) PaaS. The business model is the general application of all the software development process to the environment, such as server platform, such as computing environment as a service to provide. Cloud computing service providers will be needed for the above all kinds of development environment to provide through the Internet for software developers, and provide some additional management functions. For the software developers, on the one hand it will save the cost of buying server hardware resources such as, on the other hand through the software management function to improve the efficiency of software development. (3) SaaS. As the new software application mode, it will be the unified plan of the software in the cloud, and then through the Internet, to provide users with software and applications. The characteristics of the model is a kind of "on-demand" software, cloud service vendors provide software and hardware facilities, the user need only when demand was proposed. This pattern is largely reduced the primary cost of the software, especially significant in the use of large software costs [20].

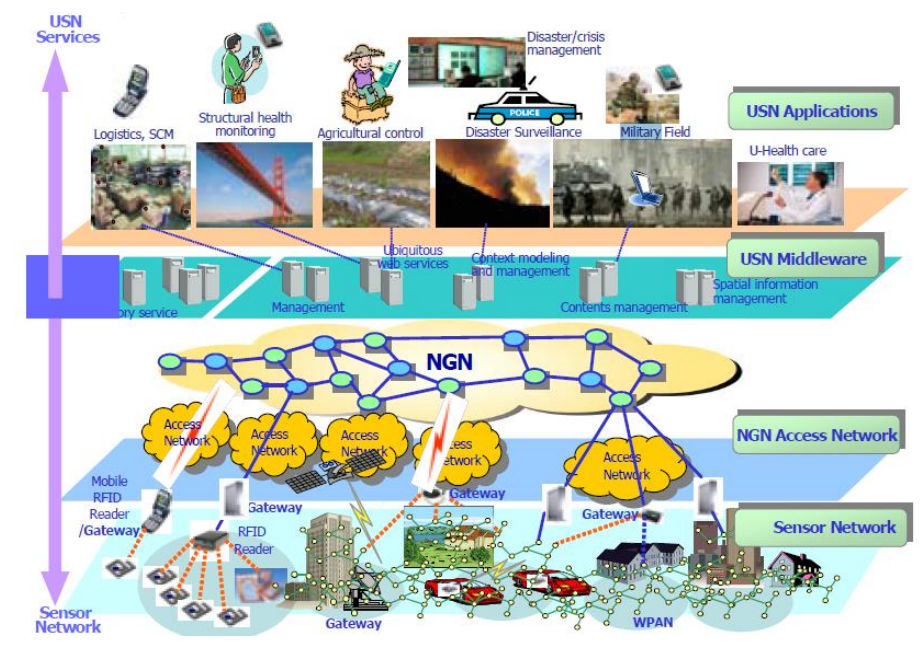

\section{Figure 4. The Concepts and Organizations of Cloud Computing Environment}

As shown in the above Figure 4, we demonstrate the concepts and organizations of cloud computing environment. Wisdom hospital platform to apply traditional users from the local number of server group migrated to the cloud platform. In this model, all the applications in the cloud automatically generated to provide users, hospital order to rent 
or to use a variety of communications services. Wisdom medical platform adopts layered architecture, a variety of applications by free combination module. It can expand or contract and the fusion application of hospital internal management system. Complete the call control and billing collection and the hospital business terminal form a complete communications office environment.

The application of cloud computing in the desktop terminal is cloud computing technology, a mature technology in the application, for the construction of medical wisdom which is the most easy to accept and popularization of an application model. Cloud terminal desktop background running on the server and the cloud storage, is a kind of by the infrastructure management layer, virtualization and the cloud platform, its appearance and widely used are described in this paper on the application of a powerful supplement which can fully improve the utilization rate of resources, cost savings and finally improve the work efficiency.

\section{The Information Management and Internet of Things}

\subsection{The Internet of Things Structures and Concepts}

Research and development of the Internet of things is still in its infancy, the domestic and foreign to the understanding of the orientation and characteristics of the Internet of things is not unified, the system model and structure of the Internet of things is not form a standard. Exist in research and development to a certain extent the exploratory, the IOT aspects such as basic research and technology development still faces many challenges. Event detection under the Internet environment, for example, and action decisions must be accurate and real time operation to ensure the accuracy of time and space in the operation of the Internet of things. Information awareness is the most basic function of the Internet of things, but through wireless sensor networks and other means to obtain the original awareness information which has significant uncertainty and a high degree of redundancy. The uncertainty of information comes from not unity, consistency, accuracy, continuity and completeness, etc. Especially among them, the heterogeneity is not unity, mainly reflected in the sensory data often have different properties, different types, different expression forms making it difficult to handle, integration and description. In the Figure 5, we show the electronic items served for IOT.

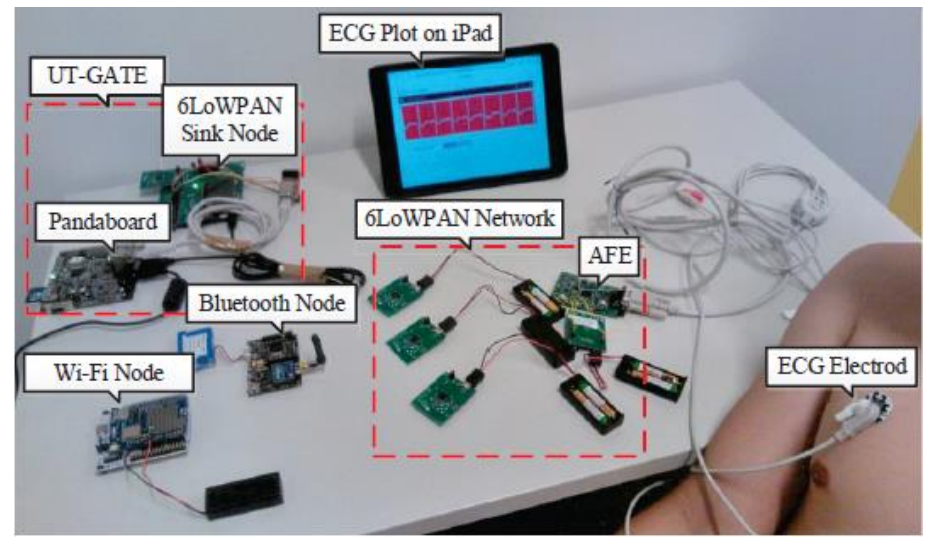

Figure 5. The Sensors and Electronics Served for the IOT System

To understand the IOT from the basic perspective, we give the definition of the technique. IOT is to integrate part of the future network and the need for standard, each other in Internet based communication protocol is the dynamic network connects of the world facilities, has a strong ability of self-configuration. In this interconnected networks, to identify all the real and virtual items need to use the particular code and through the 
seamless link of the intelligent interface, can realize the information sharing and the general access. The general IOT system is formed up with the following parts. (1) Perception layer. Perception layer at the bottom is the realization of the Internet of things to achieve the general object of information collection, the main functions of automatic identification and intelligent control in the perception layer. Another important way of obtaining information is the use of smart sensors, in the Internet of things, smart sensors can be collected and sensory information, use a variety of mechanisms to get the information expressed by some form of electrical signals and the corresponding signal processing device for processing, finally produce the corresponding action. The radio frequency identification technology is a non-contact automatic identification technology, the use of radio frequency signal automatic identification of target object, access to relevant data and this method is currently items to identify the most effective way. (2) The transport layer. IOT is located in the second layer, transport layer in the perception layer and application layer, between the main roles is to put the perception layer to obtain accurate transmission of information to the application layer, the application layer to analyze massive amounts of information, management and decision-making. The Internet of things and the transport layer can be divided into network, access network and bearing network. The IOT technique need massive information interaction wireless transmission and it is not the realistic to re-establish communication network, it need to use the core existing communication network facilities, according to the characteristics of the Internet of things to try to optimize and transform to carry all kinds of information. (3) The application layer. Key technology of Internet of things application layer includes middleware technology, cloud computing, Internet of things technologies such as business platform. The IOT middleware integration server in Internet of things and the perception layer, transport layer in the embedded devices, mainly for data proofreading of perception, collection, plays a more important role in the Internet of things. The cloud computing is based on network computing task distribution in a large number of computer constitute pool of resources, the user can use the network according to the demand for computing power with the storage space and information services [21].

From the entire medical IOT strategy, Internet of things you can enjoy church to the whole object, the process of full function and full space, the whole process of management. The above three aspects are closely linked and influence each other, so when can't solve the problem of the complete. As information heft weighs more and more important, people stress between participation in computer is the biggest of all. On the other hand, the concept of Internet of things must be in the whole process of process control. There must be a standard. In the medical industry to do the general nursery difficult standard of medical industry is not very perfect, we believe that the generation of the Internet of things based platform essentially promotes the improvement of medical information.

\subsection{The Information Management System for Healthcare Tasks}

The analysis of the medical information is a very important system. Now most of the medical record has been haphazard piled up in the reference room of the hospital, a lock is more than a decade. This is both for data storage and maintenance, more can't be use. Even if some regions have begun using electronic medical record cases, but not to summarize these valuable data, sorting, simply set up the database, make information saved in electronic form. Does not implement statistical information extraction, not to mention the late use of, make a lot of useful information resources are idle. Applying information technology to measurement and quality control of medical equipment, its essence is to use the computer implementation of medical electronic equipment measurement and quality control of information acquisition, transmission, storage, and analysis, to promote the hospital quality management of medical equipment of digital and 
intelligent. The core of the technology measurement and quality control is to establish medical equipment information management systems. However, how to obtain the original information, how to make the original information digitization is the key to build the whole system. This study attempts to take appropriate information identification technology, to build the hospital medical equipment information management system for measurement and quality control, improve the hospital quality management level.

In the following Figure 6, we illustrate the designed information management system for healthcare tasks. We could easily de-composite the system into following parts. (1) The basic system business processes. Medical equipment quality control measurement tests, using scanning electron signs read, read and acquisition equipment electronic identity number, match with the database information, to obtain information on the related equipment, and the relevant equipment information directly import measurement quality inspection record form. (2) System structure. In the medical device surface paste or electronic label, bar code reading relevant information from the reading equipment, can be automatically related equipment information collection, and then through the data transmission, sent to the computer, supply with the system call. (3) Radio frequency identification technology. On the basis of the original system requirements, from the global eyes to sort, optimization process of the system, and improve the handling of medical information, strengthen the system management concept, to implement the full electronic medical records, total cost management, total quality management of a new generation of hospital information management system. Hospital integrated management information system is built, changed the information department closed or the general state of information transmission lag, the height of the hospital internal information sharing. A complete set of RFID system, is by the reader and electronic tag and application software system is made up by three parts, its working principle is the reader to launch a specific frequency radio waves energy to electronic tags, used to drive the electronic circuit inside the tag data is sent out, the reader and interpretation in order to receive from the label of carrier signal and the received signal after demodulation and decoding to the various application system for the processing steps.

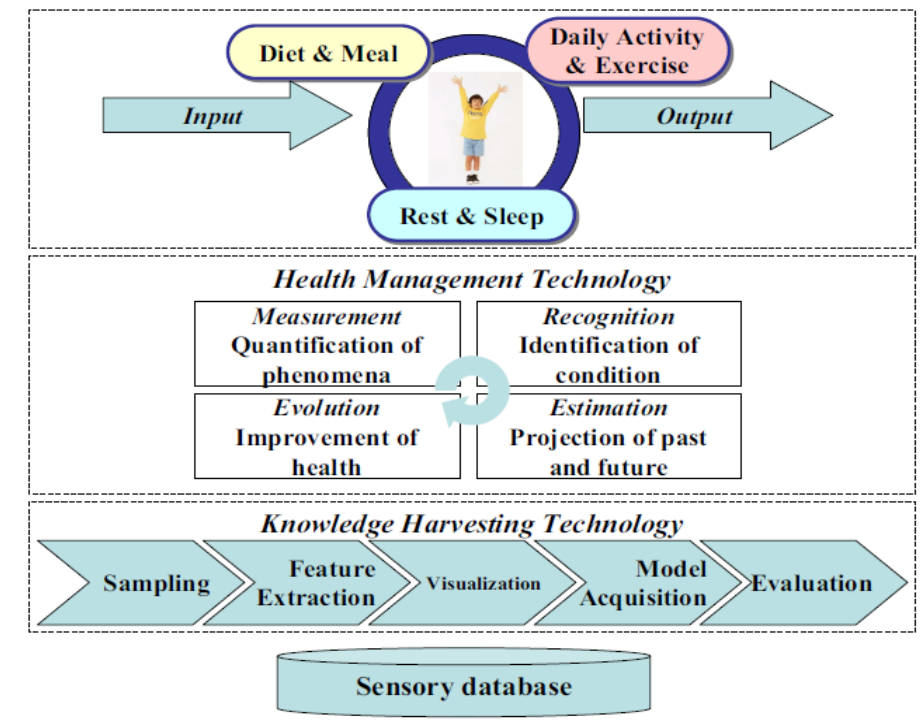

Figure 6. The Designed Information Management System for the Healthcare Tasks 


\section{The Experimental and Numerical Analysis}

\subsection{The Performance of the Data Analysis Technology for the System}

In this part, we simulate the proposed data analysis method. In the Figure 7, we show the data structure and pattern for the system. In the Figure 8, we demonstrate the data distribution after the process of the system. In the Figure 9, we illustrate the data security enhancement system organization. In the Figure 10, we show the performance of the methodology.
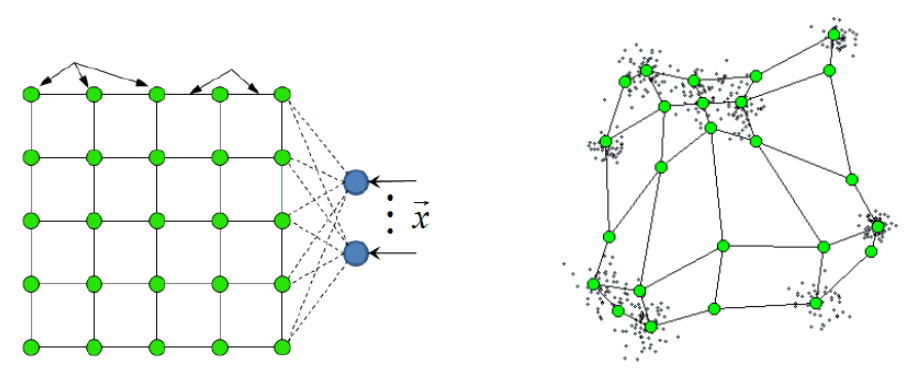

Figure 7. The Data Structure and Pattern for the System
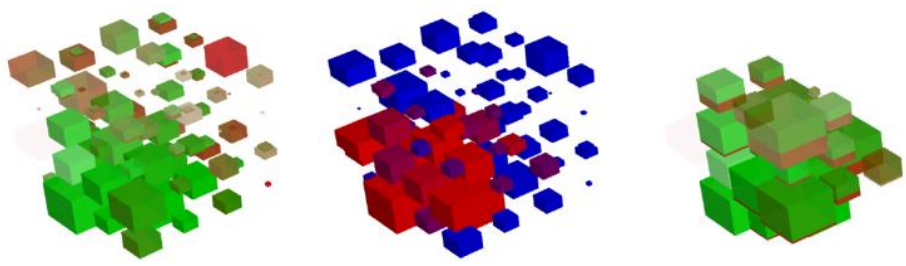

Figure 8. The Data Distribution after the Process of the System

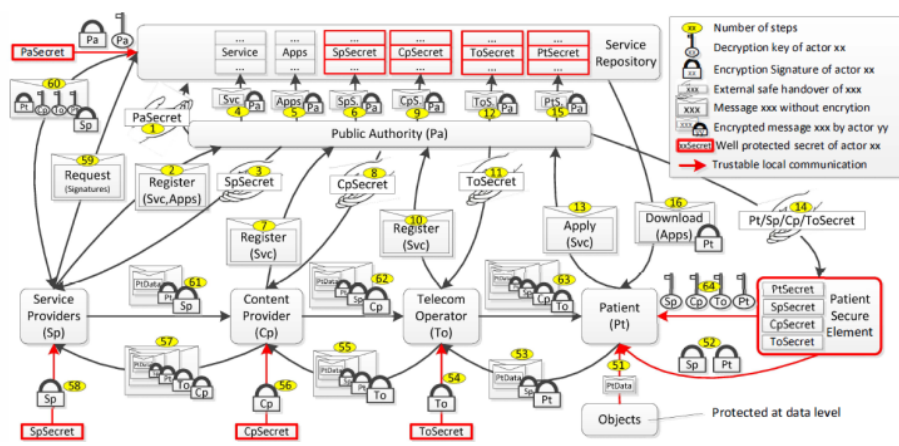

Figure 9. The Data Security Enhancement System Organization 


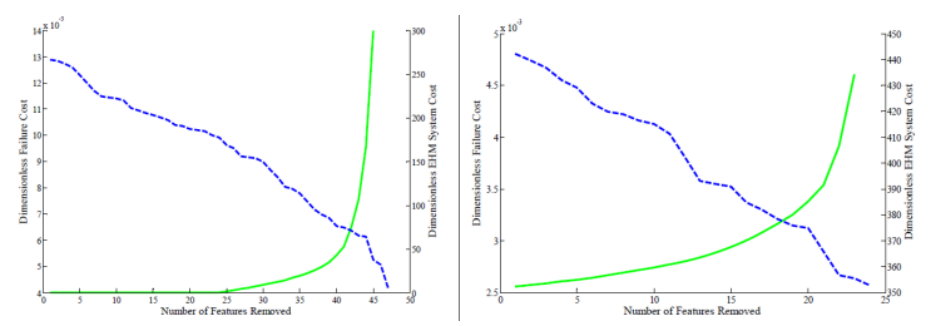

Figure 10. The Performance of the Data Analysis for the System

\subsection{The Proposed Topology of the Healthcare System}

In this section, we illustrate the structure of the designed intelligent healthcare platform. Medical wisdom is through creating healthy archives of regional health information platform, using the most advanced Internet technology, realizes the patients and the medical staff, the interaction between the medical institutions and medical equipment, gradually achieve the information. In the near future, the health care industry will melt more artificial intelligence, sensor technology and other high-tech, the medical service to the real meaning of intelligent and promote the prosperity and development of the medical enterprise. In the following Figure 11, we show the architecture of the designed intelligent healthcare platform.

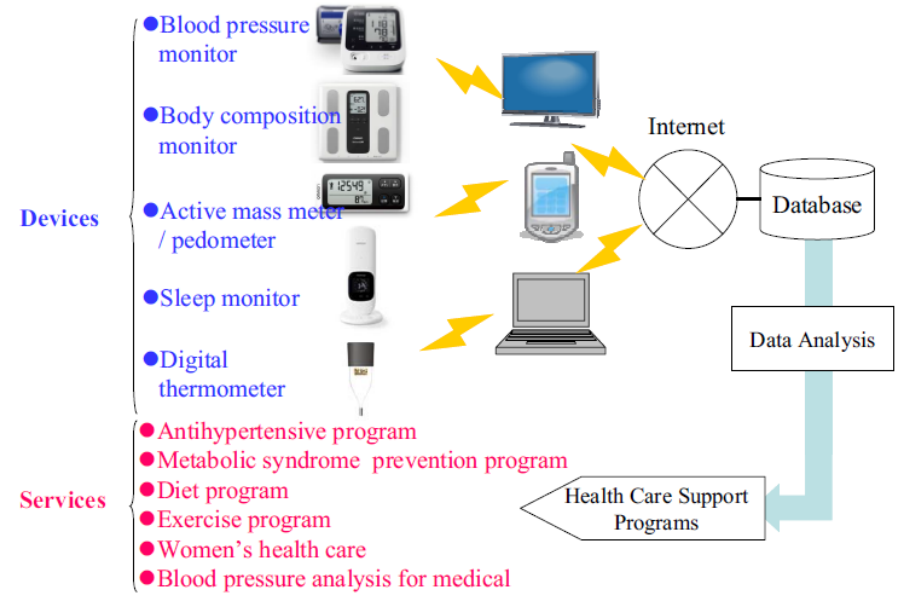

Figure 11. The Designed Intelligent Healthcare Platform

\section{Conclusion and Summary}

In this paper, we research on modern intelligent healthcare platform from the perspectives of grid based cloud computing and information management system assisted Internet of things technology. Hospital information system is set up to assist the hospital staff to process information system, clinical applications can provide fast and reliable data for the medical staff support to ensure smooth operation medical decision-making, implementation and application in the management which could provide data support for long-term period management plan. Hospital information system mainly includes two major parts of hardware system and software system, which include one or several strong center server performance, large capacity storage device and user terminal device, data communication circuit, etc., the latter including multi-function, can be more general user terminal platform software system, the system software, application software, 
development tools, information database and its management system, etc. Through the medical information management system has realized the computer management to replace artificial hand written before, the medical career with age. Medical management system mainly solves the following problems: use the keyboard instead of a record pen, computer retrieval instead of artificial from a large number of medical record text search, medical data with a computer to replace the human brain medical data calculation. Our research serves as the paradigm for the modern healthcare pattern which will enhance the current medical and healthcare mode and plays vital role in information system.

\section{References}

[1] S. S. M. Srinivasan and N. Sivagamy, "Parallel computing based intelligent web service for healthcare platform", Information Communication and Embedded Systems (ICICES), 2014 International Conference on. IEEE, (2014).

[2] G. Yang, "A Health-IoT Platform Based on the Integration of Intelligent Packaging, Unobtrusive BioSensor, and Intelligent Medicine Box", Industrial Informatics, IEEE Transactions on, vol. 10, no. 4, (2014), pp. 2180-2191.

[3] C. J. Su and C. Y. Chiang, "IAServ: An intelligent home care web services platform in a cloud for aging-in-place", International journal of environmental research and public health, vol. 10, no. 11, (2013), pp. 6106-6130.

[4] Y. Kovalchuk, K. D. McDonald-Maier and G. Howells, "Overview of ICmetrics Technology-Security Infrastructure for Autonomous and Intelligent Healthcare System." International Journal of u-and eService, Science and Technology, vol. 4, no. 3, (2011), pp. 49-60.

[5] Su, Chuan-Jun and Chia-Ying Wu, "JADE implemented mobile multi-agent based, distributed information platform for pervasive health care monitoring." Applied Soft Computing 11.1 (2011): 315325.

[6] Yang, Chao-Tung, Chi-Jui Liao, Jung-Chun Liu, Walter Den, Ying-Chyi Chou and Jaw-Ji Tsai, "Construction and application of an intelligent air quality monitoring system for healthcare environment." Journal of medical systems 38, no. 2 (2014): 1-10.

[7] Bielskis, A. A., V. Denisovas, D. Drungilas, G. Gricius, and O. Ramašauskas. "Modelling of intelligent multi-agent based E-Health care system for people with movement disabilities." Elektronika ir elektrotechnika 86, no. 6 (2015): 37-42.

[8] Ng, Kenney, Amol Ghoting, Steven R. Steinhubl, Walter F. Stewart, Bradley Malin, and Jimeng Sun. "PARAMO: A PARAllel predictive MOdeling platform for healthcare analytic research using electronic health records." Journal of biomedical informatics 48 (2014): 160-170.

[9] Wang, Pengwei, Zhijun Ding, Changjun Jiang, and Mengchu Zhou. "Web service composition techniques in a health care service platform." In Web Services (ICWS), 2011 IEEE International Conference on, pp. 355-362. IEEE, (2011).

[10] Ongenae, Femke, Maxim Claeys, Thomas Dupont, Wannes Kerckhove, Piet Verhoeve, Tom Dhaene, and Filip De Turck. "A probabilistic ontology-based platform for self-learning context-aware healthcare applications." Expert Systems With Applications 40, no. 18 (2013): 7629-7646.

[11] Datta, Chandan, Hong Yul Yang, I-Han Kuo, Elizabeth Broadbent, and Bruce A. MacDonald. "Software platform design for personal service robots in healthcare." In Robotics, Automation and Mechatronics (RAM), 2013 6th IEEE Conference on, pp. 156-161. IEEE, (2013).

[12] Bhunia, Suman Sankar, Sagar K. Dhar, and Nandini Mukherjee. "iHealth: A Fuzzy approach for provisioning Intelligent Health-care system in Smart City." In Wireless and Mobile Computing, Networking and Communications (WiMob), 2014 IEEE 10th International Conference on, pp. 187-193. IEEE, (2014).

[13] Andrès, E., S. Talha, M. Hajjam, J. Hajjam, S. Ervé, and A. Hajjam. "E-care project: a promising eplatform for optimizing management of chronic heart failure and other chronic diseases." Heart Res Open J 2, no. 1 (2015): 39-45.

[14] Chen, Min, Yujun Ma, Sana Ullah, Wei Cai, and Enmin Song. "ROCHAS: robotics and cloud-assisted healthcare system for empty nester." In Proceedings of the 8th international conference on body area networks, pp. 217-220. ICST (Institute for Computer Sciences, Social-Informatics and Telecommunications Engineering), (2013).

[15] Megalingam, Rajesh Kannan, Vineeth Radhakrishnan, Denny Chakko Jacob, Deepak Krishnan Melepurath Unnikrishnan, and Akhil Kakkanattu Sudhakaran. "Assistive technology for elders: Wireless intelligent healthcare gadget." In Global Humanitarian Technology Conference (GHTC), 2011 IEEE, pp. 296-300. IEEE, (2011).

[16] Memon, Mukhtiar, Stefan Rahr Wagner, Christian Fischer Pedersen, Femina Hassan Aysha Beevi, and Finn Overgaard Hansen. "Ambient assisted living healthcare frameworks, platforms, standards, and quality attributes." Sensors 14, no. 3 (2014): 4312-4341. 
[17] Cardoso de Moraes, João Luís, Wanderley Lopes de Souza, Luis Ferreira Pires, Luciana Tricai Cavalini, and Antonio Francisco Do Prado. "A novel architecture for message exchange in pervasive healthcare based on the use of intelligent agents." In Computer Systems and Applications (AICCSA), 2013 ACS International Conference on, pp. 1-8. IEEE, (2013).

[18] GarcíA-SáNchez, Pablo, JesúS GonzáLez, Antonio M. Mora, and Alberto Prieto. "Deploying intelligent e-health services in a mobile gateway." Expert Systems with Applications 40, no. 4 (2013): 1231-1239.

[19] Mehfuz, Shabana, Shabana Urooj, and Shivaji Sinha. "Wireless Body Area Networks: A Review with Intelligent Sensor Network-Based Emerging Technology." In Information Systems Design and Intelligent Applications, pp. 813-821. Springer India, (2015).

[20] Chen, Y-C., Y-S. Yu, R-S. Chen, I-Hsin Chou, H. M. Sun, and K. C. Yeh. "Intelligent Radioactive Waste Management Platform for Radioactive Waste Storage Facilities." Nuclear Technology 182, no. 3 (2013): 358-368.

[21] Lei, Wai Kei, Ming Chui Dong, Wei Xuan Fang, and Xiang Yang Hu. "An approach towards realizing the intelligent sphygmogram sampling for e-home healthcare." Computer methods and programs in biomedicine 108, no. 3 (2012): 1199-1205. 
International Journal of Grid and Distributed Computing Vol. 9, No. 11 (2016) 